\title{
Stochastic Analysis of a Repairable Cold Standby System Attacked by Poisson Shocks Considering Inspection and Post Repair
}

\author{
M.A.W. Mahmoud \\ Department of Mathematics, \\ Faculty of Science, \\ Al-Azhar University, \\ Nasr City (11884), \\ Cairo, Egypt.
}

\author{
A.M. Rashad \\ Department of Mathematics, \\ Faculty of Science, \\ Helwan University, \\ Helwan, \\ Cairo, Egypt
}

\author{
Z.M. Hussien \\ Department of Mathematics, \\ Faculty of Science, \\ Helwan University, \\ Helwan, \\ Cairo, Egypt
}

\begin{abstract}
This paper investigated the stochastic analysis of twodissimilar unit cold standby system considering repair, inspection, post repair under Poisson shocks. The serverman, is called when the operative unit fails. The shocks can attack the operative unit. The repaired unit is sent for inspection to decide whether the repair is satisfactory. If the repair is found unsatisfactory, then the unit is again sent for post repair. Some reliability measures of the system such as system reliability, mean time to system failure (MTSF) and steady state availability are derived. Graphical representations are presented to illustrate the theoretical results.
\end{abstract}

\section{General Terms}

Applied stochastic processes

\section{Keywords}

Poisson shock, cold standby system, mean to system failure, steady state availability.

\section{INTRODUCTION}

Reliability theory is one of the most important branches of operations research and systems engineering. Any systems analysis in order to be complete, must give due consideration to system reliability. With remarkable advances made in electronics engineering, military and communication systems have become more sophisticated and when such systems fail, very serious situations arise. Thus in the present day context, high system reliability has become very important from the view point of both makers and the users.

The shock model has been extensively in the past. For example Qingtai Wu [11] studied the reliability analysis of a cold standby system attacked by shocks. A repairable system with an unreliable repair facility and one repairman who can take single vacation considered by Renbin et al.[5]. Abdul Ameer Al-Ali and Murari, K. [1] Developed a reliability model of a single unit system with the impact of random shocks. Haitao Liu, Xianyun Meng and Wenjuan Wu [6] Considered the cold standby sytem with repair of non-new and repairman vacation. [7] Considered a cold standby repairable deteriorating system consisting of three dissimilar components and one repairman. The geometric process, and the supplementary variable techniques, a group of partial differential equations of the system were presented, and other reliability indices are obtained.[2] analyzed some reliability indices of a cold standby system with an unreliable repair facility and one repairman who can take vacation under
Poisson Shocks. By using the geometric process theory, the supplementary variable method and Laplace transform tool, availability and reliability of the system and other reliability indices are obtained. [9] considered a simple repairable system with a warning device and a repairman who can have delayed-multiple vacations. The asymptotic stability, especially the exponential stability of the system dynamic solution, is studied by using the strongly continuous semi group theory or $\mathrm{C} 0$ semi group theory. [3] discussed a singleunit system subject to random shocks. The impact of shocks may or may not be affected on this system. The single server who visits this system immediately to conduct maintenance and repair of the unit. [4] discussed the effect of human failures on the reliability of the system and determine the cost function, and some reliability indices were derived. [10] analyzed some reliability indices of a cold standby system consisting of two repairable units, a switch and a repairman who may not always be at the job site or take vacation. [12] studied a k-out-of-n : G system and a consecutive-k-out-ofn : $\mathrm{F}$ system, respectively, with $\mathrm{R}$ repairmen who can take multiple vacations and by using Markov model; the analytically solution of some reliability indices was discussed. [14] studied a deteriorating system with a repairman who can have multiple vacations. By means of the geometric process and the supplementary variable techniques, a group of partial differential equations of the system was presented, and some reliability indices were derived. [13] considered a deteriorating repairable system and a cold standby repairable system with two different components of different priority in use, both with one repairman who can take multiple vacations. The explicit expression of the expected cost rate was given, and an optimal replacement policy was discussed. [8] deal with the study of the stochastic analysis of a two-unit cold standby system consid ering hardware failure, human error failure and preventive maintenance.

The present paper, we consider a two-unit cold standby (nonidentical) with inspection time under Poisson shocks. The arrival time of the shocks follow a homogeneous Poisson process and other distribution are arbitrary distribution. Finally, the effects of parameters on the system performance have been studied. 


\section{MODEL DESCRIPTION AND ASSUMPTIONS}

1. The system consists of two non-identical units. Initially one unit is operating and the other is in standby case (cold standby).

2. The switch is perfect and instantaneously.

3. The system is subject to shocks. The arrivals of the shocks follow a Poisson process $[N(t), t \geq 0]$ with the intensity $\lambda>0$. The magnitude of each shock $\hat{X}$, is a random variable with distribution function $F$.

4. When a shock arrives, it only affects the operating unit. The operating unit will fail when the magnitude of a shock exceeds a threshold. The threshold of unit $i$ is a non-negative random variable $\tau_{i}$ with a distribution function $\Phi_{i}(i=1,2)$.

5. The repairman already is unavailable when two units are good. He is demanded when the failure occurs. If a unit fails when the other is being repaired, the newly failed unit must wait for repair and the system is down. If two units are waiting for repair when the repairman comes to the system, unit 1 has the priority to be repaired.

6. After the repair, a unit goes for inspection to decide whether the repair is satisfactory or not. If the repaired unit is found to be unsatisfactory then it is sent for post repair. The probability of having satisfactory repair is fixed.

7. Service discipline is a first-come, first-served (FCFS). A single perfect repair facility is available for repair, inspection and post repair.

8. Once the repairman completes his work, he leaves the system.

9. Shocks are assumed to be only cause of unit failure, and the system fails when both the units fail.

10. The repair, inspection, post repair and the demanding (waiting) time are assumed to be arbitrary.

11. All random variables are independent. At the beginning, the two units are new, one unit starts to work, the other unit is on cold standby and the repairman goes out the system. The units can be repaired as good as new.

\section{NOTATIONS}

\begin{tabular}{|l|l|}
\hline$H_{i}(i=1,2)$ & $\begin{array}{l}\text { As unit's repair time. Their distributions are } \\
\left\{\bar{H}_{i}(t)=e^{-\int_{0}^{t} \mu_{i}\left(y_{i}\right) d y_{i}}\right\} ; \text { where } \mu_{i}\left(y_{i}\right) \text { is } \\
\text { hazard rate function. }\end{array}$ \\
\hline $\mathrm{V}$ & $\begin{array}{l}\text { As demanding (waiting) time. Its distribution } \\
\text { is }\left\{\bar{V}(t)=e^{-\int_{0}^{t} \alpha(x) d x}\right\} ; \text { where } \alpha(x) \text { is hazard } \\
\text { rate function. }\end{array}$ \\
\hline $\mathrm{A}$ & $\begin{array}{l}\text { As the inspection time. Its distribution is } \\
\left\{\bar{A}(t)=e^{-\int_{0}^{t} \gamma(z) d z}\right\} ; \text { where } \gamma(z) \text { is hazard } \\
\text { rate function. }\end{array}$ \\
\hline$U_{i}(i=1,2)$ & $\begin{array}{l}\text { As post repair time. Their distributions are }\{ \\
\left.\overline{\mathrm{U}}_{\mathrm{i}}(\mathrm{t})=\mathrm{e}^{-\int_{0}^{t} \mathrm{k}_{\mathrm{i}}\left(\mathrm{t}_{\mathrm{i}}\right) \mathrm{d} \mathrm{t}_{\mathrm{i}}}\right\} ; \text { where } \mathrm{k}_{\mathrm{i}}\left(\mathrm{t}_{\mathrm{i}}\right) \text { is hazard } \\
\text { rate function. }\end{array}$ \\
\hline$*$ & $\begin{array}{l}\text { Laplace transforms; }\left\{f^{*}(s)=L_{s}[f(x)]\right. \\
\left.=\int_{0}^{\infty} f(x) e^{-x s} d x ; s>0\right\} .\end{array}$ \\
\hline $\mathrm{q}$ & $\begin{array}{l}\text { The probability that the repaired unit is } \\
\text { unsatisfactory. }\end{array}$ \\
\hline
\end{tabular}

\begin{tabular}{|l|l|}
\hline AV & The steady-state availability of system. \\
\hline $\mathrm{R}(\mathrm{t})$ & The reliability of the system. \\
\hline MTTF & Mean time to the system failure. \\
\hline$r_{i}$ & $\begin{array}{l}\text { The probability that one shock causes unit } i \text { to } \\
\text { fail, }(i=1,2)\end{array}$ \\
\hline
\end{tabular}

\section{SYSTEM ANALYSIS}

With the model assumptions given in the preceding section, the failure probability of unit $i(i=1,2)$, given the shock value $\hat{x}$, is $\Phi_{i}(\hat{x})=P(\tau<\hat{x})$. Since the magnitude of a shock is a random variable $\hat{X}$, the conditional failure probability of unit $i$ is a random $\Phi_{i}(\hat{x})$ with $(i=1,2)$, respectively, and its probability distribution can be written by: $P_{i}(x)=$ $P\left(\Phi_{i}(\hat{X}) \leq x\right)=P\left(\hat{X} \leq \Phi_{i}{ }^{-1}(x)\right)=F\left(\Phi_{i}{ }^{-1}(x)\right), 0<$ $x<1,(i=1,2)$. From assumption 2 and 3 , we can see that, the probability that one shock causes unit $i$ to fail is:

$$
\begin{aligned}
r_{i} & =P\left(\hat{X}>\tau_{i}\right) \\
& =\int_{0}^{\infty} P\left(\tau_{i}<\hat{x} \mid \hat{X}=\hat{x}\right) d P(\hat{X} \leq \hat{x})=\int_{0}^{\infty} \Phi_{i}(\hat{x}) d F(\hat{x}) . \\
& (i=1,2) .
\end{aligned}
$$

Let $S(t)$ be the system state at time t, then

State 0 : unit 1 is working and unit 2 is on cold standby.

State 1: unit 2 is working, unit 1 is waiting for repair and the repairman is calling.

State 2: unit 2 is working, unit 1 is being repaired.

State 3: the two units are waiting for repair because of the repairman does come.

State 4: unit 2 is working, unit 1 is being inspected.

State 5: unit 1 is under repair and unit 2 is waiting for repair.

State 6: unit 2 is working while unit 1 is being cold standby.

State 7: unit 2 is working and unit 1 is being post repaired.

State 8: unit 1 is being inspected and unit 2 is waiting for repair.

State9: unit 1 is being post repaired and unit 2 is waiting for repair.

State 10: unit 1 is working and unit 2 is being repaired.

State 11: unit 1 is working, unit 2 is waiting for repair and the repairman is calling.

State 12: unit 2 is under repair; unit 1 is waiting for repair.

State 13: unit 1 is working and unit 2 is being inspected.

State 14: unit 1 is working and unit 2 is being post repaired.

State 15: unit 2 is being inspected and unit 1 is waiting for repair.

State16: unit 2 is being post repaired and unit 1 is waiting for repair.

The state space is

$\Omega=\{0,1,2,3,4,5,6,7,8,9,10,11,12,13,14,15,16\}$, where the up state set is $W=\{0,1,2,4,6,7,10,11,13,14\}$ and the down state set $\mathrm{D}=\{3,5,8,9,12,15,16\}$.

One can note that $\{S(t), t \geq 0\}$ is not a Markov process, where $S(t)$ represents all states of the system. So we introduce supplementary variable:

i). $\mathrm{X}(\mathrm{t})$ : if $S(t)=1,3,11$.Then $\mathrm{X}(\mathrm{t})$ is the elapsed summoned time when the repairman is not exist at time $t$.

ii). $\mathrm{Y}_{1}(\mathrm{t})$ : if $S(t)=2,5$. Then $\mathrm{Y}_{1}(\mathrm{t})$ is the elapsed repair time of unit 1 being repaired at time $t$.

iii). $\mathrm{Y}_{2}(\mathrm{t})$ : if $S(t)=10,12$. Then $\mathrm{Y}_{2}(\mathrm{t})$ is the elapsed repair time of unit 2 being repaired at time $t$

iv). $\mathrm{Z}(\mathrm{t})$ if $S(t)=4,8,13,15$. Then $\mathrm{Z}(\mathrm{t})$ is the elapsed inspection time at time $t$. 
v). $\mathrm{U}_{1}(\mathrm{t})$ : if $S(t)=7,9$. Then $\mathrm{U}_{1}(\mathrm{t})$ is the elapsed post repair time of unit 1 being post repaired at time t.

vi). $\mathrm{U}_{2}(\mathrm{t})$ : if $S(t)=14,16$. Then $\mathrm{U}_{2}(\mathrm{t})$ is the elapsed post repair time of unit 2 being post repaired at time $t$

Then $\left\{\left(S(t), X(t), \mathrm{Y}_{1}(\mathrm{t}), \mathrm{Y}_{2}(\mathrm{t}), \mathrm{Z}(\mathrm{t}), \mathrm{U}_{1}(\mathrm{t}), \mathrm{U}_{2}(\mathrm{t})\right), \mathrm{t} \geq 0\right\}$ is a generalized Markov process. let:

$$
\begin{array}{ll}
Q_{i}(t, x)=P(S(t)=i, X(t) \leq x), & (i=1,3,11) . \\
Q_{i}\left(t, y_{1}\right)=P\left(S(t)=i, Y_{1}(t) \leq y_{1}\right), & (i=2,5) . \\
Q_{i}\left(t, y_{2}\right)=P\left(S(t)=i, Y_{2}(t) \leq y_{2}\right), & (i=10,12) \\
Q_{i}(t, z)=P(S(t)=i, Z(t) \leq z), & (i=4,8,13,15) . \\
Q_{i}\left(t, u_{1}\right)=P\left(S(t)=i, U_{1}(t) \leq u_{1}\right), & (i=7,9) .
\end{array}
$$

and,

$Q_{i}\left(t, u_{2}\right)=P\left(S(t)=i, U_{2}(t) \leq u_{2}\right) . \quad(i=14,16)$.

where $P(B)$ is probability of event $\mathrm{B}$, consider:

$P_{i}(t, w)=\frac{d}{d w} Q_{i}(t, w)$

$$
;(i=\{1,2,3,4,5,7,8,9,10,11,12,13,14,15,16\}) .
$$

We can express the process in a way considering the transitions in $t$ and $t+\Delta t$.

It is easily to show that,

$$
\begin{aligned}
P_{0}(t+\Delta t)= & P_{0}(t)\left(1-r_{1} \lambda \Delta t\right)+\int_{0}^{\infty} k_{2}\left(u_{2}\right) P_{14}\left(t, u_{2}\right) d u_{2} \Delta t \\
& +\int_{0}^{\infty}(1-q) \gamma(z) P_{13}(t, z) d z \Delta t+o(\Delta t)
\end{aligned}
$$

then,

$P_{0}(t+\Delta t)-P_{0}(t)=-r_{1} \lambda \Delta t P_{0}(t)+\int_{0}^{\infty} k_{2}\left(u_{2}\right) P_{14}\left(t, u_{2}\right) d u_{2} \Delta t$

$$
+\int_{0}^{\infty}(1-q) \gamma(z) P_{13}(t, z) d z \Delta t+o(\Delta t) .
$$

Dividing both sides of (2) .we get,

$$
\begin{aligned}
\lim _{\Delta t \rightarrow 0} \frac{P_{0}(t+\Delta t)-}{\Delta t} & P_{0}(t) \\
& =-r_{1} \lambda P_{0}(t)+\int_{0}^{\infty} k_{2}\left(u_{2}\right) P_{14}\left(t, u_{2}\right) d u_{2} \\
& +\int_{0}^{\infty}(1-q) \gamma(z) P_{13}(t, z) d z,
\end{aligned}
$$

and this yields

$$
\begin{aligned}
\left(\frac{d}{d t}\right. & \left.+r_{1} \lambda\right) P_{0}(t)= \\
& =\int_{0}^{\infty} k_{2}\left(u_{2}\right) P_{14}\left(t, u_{2}\right) d u_{2}+\int_{0}^{\infty}(1-q) \gamma(z) P_{13}(t, z) d z
\end{aligned}
$$

By the same arguments, the following partial-differential equations can be obtained

$$
\begin{aligned}
& \left(\frac{\partial}{\partial t}+\frac{\partial}{\partial x}+\alpha(x)+r_{2} \lambda\right) P_{1}(t, x)=0 \\
& \left(\frac{\partial}{\partial t}+\frac{\partial}{\partial y_{1}}+\mu_{1}\left(y_{1}\right)+r_{2} \lambda\right) P_{2}\left(t, y_{1}\right)=0 \\
& \left(\frac{\partial}{\partial t}+\frac{\partial}{\partial x}+\alpha(x)\right) P_{3}(t, x)=r_{2} \lambda P_{1}(t, x)+r_{1} \lambda P_{11}(t, x), \\
& \left(\frac{\partial}{\partial t}+\frac{\partial}{\partial z}+\gamma(z)+r_{2} \lambda\right) P_{4}(t, z)=0
\end{aligned}
$$

$$
\begin{aligned}
& \left(\frac{\partial}{\partial t}+\frac{\partial}{\partial y_{1}}+\mu_{1}\left(y_{1}\right)\right) P_{5}\left(t, y_{1}\right)=r_{2} \lambda P_{2}\left(t, y_{1}\right), \\
& \left(\frac{d}{d t}+r_{2} \lambda\right) P_{6}(t)=\int_{0}^{\infty} k_{1}\left(u_{1}\right) P_{7}\left(t, u_{1}\right) d u_{1} \\
& \quad+\int_{0}^{\infty}(1-q) \gamma(z) P_{4}(t, z) d z, \\
& \left(\frac{\partial}{\partial t}+\frac{\partial}{\partial u_{1}}+k_{1}\left(u_{1}\right)+r_{2} \lambda\right) P_{7}\left(t, u_{1}\right)=0, \\
& \left(\frac{\partial}{\partial t}+\frac{\partial}{\partial z}+\gamma(z)\right) P_{8}(t, z)=r_{2} \lambda P_{4}(t, z), \\
& \left(\frac{\partial}{\partial t}+\frac{\partial}{\partial u_{1}}+k_{1}\left(u_{1}\right)\right) P_{9}\left(t, u_{1}\right)=r_{2} \lambda P_{7}\left(t, u_{1}\right), \\
& \left(\frac{\partial}{\partial t}+\frac{\partial}{\partial y_{2}}+\mu_{2}\left(y_{2}\right)+r_{1} \lambda\right) P_{10}\left(t, y_{2}\right)=0, \\
& \left(\frac{\partial}{\partial t}+\frac{\partial}{\partial x}+\alpha(x)+r_{1} \lambda\right) P_{11}(t, x)=0, \\
& \left(\frac{\partial}{\partial t}+\frac{\partial}{\partial y_{2}}+\mu_{2}\left(y_{2}\right)+r_{1} \lambda\right) P_{12}\left(t, y_{2}\right)=r_{1} \lambda P_{10}\left(t, y_{2}\right), \\
& \left(\frac{\partial}{\partial t}+\frac{\partial}{\partial z}+\gamma(z)+r_{1} \lambda\right) P_{13}(t, z)=0, \\
& \left(\frac{\partial}{\partial t}+\frac{\partial}{\partial u_{2}}+k_{2}\left(u_{2}\right)+r_{1} \lambda\right) P_{14}\left(t, u_{2}\right)=0, \\
& \left(\frac{\partial}{\partial t}+\frac{\partial}{\partial z}+\gamma(z)\right) P_{15}(t, z)=r_{1} \lambda P_{13}(t, z),
\end{aligned}
$$

and

$\left(\frac{\partial}{\partial t}+\frac{\partial}{\partial u_{2}}+k_{2}\left(u_{2}\right)\right) P_{16}\left(t, u_{2}\right)=r_{1} \lambda P_{14}\left(t, u_{2}\right)$.

Their boundary conditions are:-

$P_{1}(t, 0)=r_{1} \lambda P_{0}(t)+\delta(t), \quad$ where, $\delta(t)= \begin{cases}1, & t=0 \\ 0, & t \neq 0\end{cases}$

$$
\begin{aligned}
& P_{2}(t, 0)= \int_{0}^{\infty} \alpha(x) P_{1}(t, x) d x+\int_{0}^{\infty} k_{2}\left(u_{2}\right) P_{16}\left(t, u_{2}\right) d u_{2} \\
&+\int_{0}^{\infty}(1-q) \gamma(z) P_{15}(t, z) d z \\
& P_{3}(t, 0)= P_{12}(t, 0)=0 \\
& P_{4}(t, 0)= \int_{0}^{\infty} \mu_{1}\left(y_{1}\right) P_{2}\left(t, y_{1}\right) d y_{1} \\
& P_{5}(t, 0)= \int_{0}^{\infty} \alpha(x) P_{3}(t, x) d x \\
& P_{7}(t, 0)= \int_{0}^{\infty} q \gamma(z) P_{4}(t, z) d z \\
& P_{8}(t, 0)= \int_{0}^{\infty} \mu_{1}\left(y_{1}\right) P_{5}\left(t, y_{1}\right) d y_{1} \\
& P_{9}(t, 0)= \int_{0}^{\infty} q \gamma(z) P_{8}(t, z) d z \\
& P_{10}(t, 0)= \int_{0}^{\infty} \alpha(x) P_{11}(t, x) d x+\int_{0}^{\infty} k_{1}\left(u_{1}\right) P_{9}\left(t, u_{1}\right) d u_{1} \\
&+\int_{0}^{\infty}(1-q) \gamma(z) P_{8}(t, z) d z \\
& P_{11}(t, 0)= r_{2} \lambda P_{6}(t), \\
& P_{13}(t, 0)= \int_{0}^{\infty} \mu_{2}\left(y_{2}\right) P_{10}\left(t, y_{2}\right) d y_{2} \\
& P_{14}(t, 0)= \int_{0}^{\infty} q \gamma(z) P_{13}(t, z) d z \\
& P_{15}(t, 0)= \int_{0}^{\infty} \mu_{2}\left(y_{2}\right) P_{12}\left(t, y_{2}\right) d y_{2} \\
& \text { and },
\end{aligned}
$$


$P_{16}(t, 0)=\int_{0}^{\infty} q \gamma(z) P_{15}(t, z) d z$.

The initial conditions are

$P_{1}(0, x)=\delta(x)=\left\{\begin{array}{ll}1, & x=0 \\ 0, & x \neq 0\end{array}\right.$,

Otherwise is 0 .

It is noticed that:

$P_{0}(t)+\sum_{i=1}^{5} \int_{0}^{\infty} P_{i}(t, x) d x+P_{6}(t)+\sum_{j=7}^{16} \int_{0}^{\infty} P_{j}(t, x) d x=1$.

\section{THE STEADY-STATE AVAILABILITY NORMAL}

Define,

$P_{i}=\lim _{t \rightarrow \infty} P_{i}(t)$

$$
\text { ,i }=\{0,1,2, \ldots, 16\},
$$

and,

$g_{i}(u)=\lim _{t \rightarrow \infty} P_{i}(t, u)$

$$
; i=\{1,2,3,4,5,7,8,9,10,11,12,13,14,15,16\} .
$$

This follows the following relations:

$P_{i}=\int_{0}^{\infty} g_{i}(u) d u$

$$
\text { ; }(\mathrm{i}=\{1,2,3,4,5,7,8,9,10,11,12,13,14,15,16\})
$$

After taking the, $\lim _{t \rightarrow \infty}$ for both sides of Eq.(3-33), we can obtain the following equations:

$r_{1} \lambda P_{0}=\int_{0}^{\infty} k_{2}\left(u_{2}\right) g_{14}\left(u_{2}\right) d u_{2}+\int_{0}^{\infty}(1-q) \gamma(z) g_{13}(z) d z$

(36)

$$
\begin{aligned}
& \left(\frac{d}{d x}+\alpha(x)+r_{2} \lambda\right) g_{1}(x)=0, \\
& \left(\frac{d}{d y_{1}}+\mu_{1}\left(y_{1}\right)+r_{2} \lambda\right) g_{2}\left(y_{1}\right)=0, \\
& \left(\frac{d}{d x}+\alpha(x)\right) g_{3}(x)=r_{2} \lambda g_{1}(x)+r_{1} \lambda g_{11}(x), \\
& \left(\frac{d}{d z}+\gamma(z)+r_{2} \lambda\right) g_{4}(z)=0, \\
& \left(\frac{d}{d y_{1}}+\mu_{1}\left(y_{1}\right)\right) g_{5}\left(y_{1}\right)=r_{2} \lambda g_{2}\left(y_{1}\right),
\end{aligned}
$$

$r_{2} \lambda P_{6}=\int_{0}^{\infty} k_{1}\left(u_{1}\right) g_{7}\left(u_{1}\right) d u_{1}+\int_{0}^{\infty}(1-q) \gamma(z) g_{4}(z) d z$,

$$
\begin{aligned}
& \left(\frac{d}{d u_{1}}+k_{1}\left(u_{1}\right)+r_{2} \lambda\right) g_{7}\left(u_{1}\right)=0, \\
& \left(\frac{d}{d z}+\gamma(z)\right) g_{8}(z)=r_{2} \lambda g_{4}(z), \\
& \left(\frac{d}{d u_{1}}+k_{1}\left(u_{1}\right)\right) g_{9}\left(u_{1}\right)=r_{2} \lambda g_{7}\left(u_{1}\right), \\
& \left(\frac{d}{d y_{2}}+\mu_{2}\left(y_{2}\right)+r_{1} \lambda\right) g_{10}\left(y_{2}\right)=0, \\
& \left(\frac{d}{d x}+\alpha(x)+r_{1} \lambda\right) g_{11}(x)=0, \\
& \left(\frac{d}{d y_{2}}+\mu_{2}\left(y_{2}\right)+r_{1} \lambda\right) g_{12}\left(y_{2}\right)=r_{1} \lambda g_{10}\left(y_{2}\right), \\
& \left(\frac{d}{d z}+\gamma(z)+r_{1} \lambda\right) g_{13}(z)=0,
\end{aligned}
$$

$$
\begin{aligned}
& \left(\frac{d}{d u_{2}}+k_{2}\left(u_{2}\right)+r_{1} \lambda\right) g_{14}\left(u_{2}\right)=0, \\
& \left(\frac{d}{d z}+\gamma(z)\right) g_{15}(z)=r_{1} \lambda g_{13}(z), \\
& \left(\frac{d}{d u_{2}}+k_{2}\left(u_{2}\right)\right) g_{16}\left(u_{2}\right)=r_{1} \lambda g_{14}\left(u_{2}\right),
\end{aligned}
$$

In this case the boundary condition can be put as follows:

$g_{1}(0)=r_{1} \lambda P_{0}$,

$g_{2}(0)=\int_{0}^{\infty} \alpha(x) g_{1}(x) d x+\int_{0}^{\infty} k_{2}\left(u_{2}\right) g_{16}\left(u_{2}\right) d u_{2}$

$$
+\int_{0}^{\infty}(1-q) \gamma(z) g_{15}(z) d z
$$

$g_{3}(0)=g_{12}(0)=0$,

$g_{4}(0)=\int_{0}^{\infty} \mu_{1}\left(y_{1}\right) g_{2}\left(y_{1}\right) d y_{1}$,

$g_{5}(0)=\int_{0}^{\infty} \alpha(x) g_{3}(x) d x$,

$g_{7}(0)=\int_{0}^{\infty} q \gamma(z) g_{4}(z) d z$,

$g_{8}(0)=\int_{0}^{\infty} \mu_{1}\left(y_{1}\right) g_{5}\left(y_{1}\right) d y_{1}$,

$g_{9}(0)=\int_{0}^{\infty} q \gamma(z) g_{8}(z) d z$

$g_{10}(0)=\int_{0}^{\infty} \alpha(x) g_{11}(x) d x+\int_{0}^{\infty} k_{1}\left(u_{1}\right) g_{9}\left(, u_{1}\right) d u_{1}$ $+\int_{0}^{\infty}(1-q) \gamma(z) g_{8}(, z) d z$,

$g_{11}(0)=r_{2} \lambda P_{6}$,

$g_{13}(0)=\int_{0}^{\infty} \mu_{2}\left(y_{2}\right) g_{10}\left(y_{2}\right) d y_{2}$,

$g_{14}(0)=\int_{0}^{\infty} q \gamma(z) g_{13}(z) d z$,

$g_{15}(0)=\int_{0}^{\infty} \mu_{2}\left(y_{2}\right) g_{12}\left(y_{2}\right) d y_{2}$,

and,

$g_{16}(0)=\int_{0}^{\infty} q \gamma(z) g_{15}(z) d z$.

Solving
$P_{i}=\int_{0}^{\infty} g_{i}(u) d u$

considering

; $(\mathrm{i}=\{1,2,3,4,5,7,8,9,10,11,12,13,14,15,16\})$

We get the following steady-state probabilities:

$P_{0}=\frac{c_{0}}{\lambda r_{1}}$,

$P_{1}=c_{0} \overline{V^{*}}\left(r_{2} \lambda\right)$,

$P_{2}=c_{0}\left(-1+n+v^{*}\left[\lambda r_{2}\right]\right)\left(\bar{H}_{1}\right)^{*}\left[\lambda r_{2}\right]$,

$P_{3}=c_{0}\left(\frac{1}{\alpha}-\bar{V}^{*}\left[\lambda r_{2}\right]+a^{*}\left[\lambda r_{2}\right]\left(-1+n+v^{*}\left[\lambda r_{2}\right]\right)\left(\frac{1}{\alpha}-\right.\right.$ $\left.\left.\bar{V}^{*}\left[\lambda r_{1}\right]\right)\left(h_{1}\right)^{*}\left[\lambda r_{2}\right]\left(1-q+q\left(u_{1}\right)^{*}\left[\lambda r_{2}\right]\right)\right)$,

(70)

$P_{4}=c_{0}\left(-1+n+v^{*}\left[\lambda r_{2}\right]\right) \bar{A}^{*}\left[\lambda r_{2}\right]\left(h_{1}\right)^{*}\left[\lambda r_{2}\right]$,

$P_{5}=c_{0}\left\{\frac{1}{\mu_{1}}\left(n-a^{*}\left[\lambda r_{2}\right]\left(-1+v^{*}\left[\lambda r_{1}\right]\right)(-1+n+\right.\right.$ $\left.\left.v^{*}\left[\lambda r_{2}\right]\right)\left(h_{1}\right)^{*}\left[\lambda r_{2}\right]\left(1-q+q\left(u_{1}\right)^{*}\left[\lambda r_{2}\right]\right)\right)-(-1+n+$ $\left.\left.v^{*}\left[\lambda r_{2}\right]\right)\left(\bar{H}_{1}\right)^{*}\left[\lambda r_{2}\right]\right\}$, 
$P_{6}=\frac{c_{0}}{\lambda r_{2}}\left(a^{*}\left[\lambda r_{2}\right]\left(-1+n+v^{*}\left[\lambda r_{2}\right]\right)\left(h_{1}\right)^{*}\left[\lambda r_{2}\right](1-q+\right.$ $\left.\left.q\left(u_{1}\right)^{*}\left[\lambda r_{2}\right]\right)\right)$,

$P_{7}=c_{0} q a^{*}\left[\lambda r_{2}\right]\left(h_{1}\right)^{*}\left[\lambda r_{2}\right]\left(\bar{U}_{1}\right)^{*}\left[\lambda r_{2}\right]\left(-1+n+v^{*}\left[\lambda r_{2}\right]\right)$,

$P_{8}=\frac{1}{\gamma} c_{0}\left(n-\left(-1+n+v^{*}\left[\lambda r_{2}\right]\right)\left(h_{1}\right)^{*}\left[\lambda r_{2}\right]\left(\gamma \bar{A}^{*}\left[\lambda r_{2}\right]+\right.\right.$

$\left.\left.a^{*}\left[\lambda r_{2}\right]\left(-1+v^{*}\left[\lambda r_{1}\right]\right)\left(1-q+q\left(u_{1}\right)^{*}\left[\lambda r_{2}\right]\right)\right)\right)$,

$P_{9}=\frac{q c_{0}}{k_{2}}\left(n-a^{*}\left[\lambda r_{2}\right]\left(-1+n+v^{*}\left[\lambda r_{2}\right]\right)\left(h_{1}\right)^{*}\left[\lambda r_{2}\right]((-1+\right.$ $\left.\left.\left.v^{*}\left[\lambda r_{1}\right]\right)\left(1-q+q\left(u_{1}\right)^{*}\left[\lambda r_{2}\right]\right)+k_{2}\left(\bar{U}_{1}\right)^{*}\left[\lambda r_{2}\right]\right)\right)$,

$P_{10}=n c_{0}\left(\bar{H}_{2}\right)^{*}\left[\lambda r_{1}\right]$,

$P_{11}=c_{0} a^{*}\left[\lambda r_{2}\right]\left(-1+n+v^{*}\left[\lambda r_{2}\right]\right) \bar{V}^{*}\left[\lambda r_{1}\right]\left(h_{1}\right)^{*}\left[\lambda r_{2}\right](1-$

$\left.q+q\left(u_{1}\right)^{*}\left[\lambda r_{2}\right]\right)$,

$P_{12}=n c_{0}\left(\frac{1}{\mu_{2}}-\left(\bar{H}_{2}\right)^{*}\left[\lambda r_{1}\right]\right)$,

$P_{13}=n c_{0} \bar{A}^{*}\left[\lambda r_{1}\right]\left(h_{2}\right)^{*}\left[\lambda r_{1}\right]$,

$P_{14}=n q c_{0} a^{*}\left[\lambda r_{1}\right]\left(h_{2}\right)^{*}\left[\lambda r_{1}\right]\left(\bar{U}_{2}\right)^{*}\left[\lambda r_{1}\right]$,

$P_{15}=n c_{0}\left(\frac{1}{\gamma}-\bar{A}^{*}\left[\lambda r_{1}\right]\left(h_{2}\right)^{*}\left[\lambda r_{1}\right]\right)$,

and, $P_{16}=n q c_{0}\left(\frac{1}{k_{2}}-a^{*}\left[\lambda r_{1}\right]\left(h_{2}\right)^{*}\left[\lambda r_{1}\right]\left(\bar{U}_{2}\right)^{*}\left[\lambda r_{1}\right]\right)$.

where,

$c_{0}=\frac{\alpha \gamma \lambda k_{2} r_{1} r_{2} \mu_{1} \mu_{2}}{d}$,

$d=q \alpha \gamma \lambda r_{1} r_{2} \mu_{1} \mu_{2}\left\{2 n-a^{*}\left[\lambda r_{2}\right]\left(-1+v^{*}\left[\lambda r_{1}\right]\right)(-1+n+\right.$ $\left.\left.v^{*}\left[\lambda r_{2}\right]\right)\left(h_{1}\right)^{*}\left[\lambda r_{2}\right]\left(1-q+q\left(u_{1}\right)^{*}\left[\lambda r_{2}\right]\right)\right)+k_{2}\left(\alpha \gamma r_{2} \mu_{1} \mu_{2}+\right.$ $r_{1}\left(\alpha \gamma \mu_{1} \mu_{2} a^{*}\left[\lambda r_{2}\right]\left(-1+n+v^{*}\left[\lambda r_{2}\right]\right)\left(h_{1}\right)^{*}\left[\lambda r_{2}\right](1-q+\right.$ $\left.q\left(u_{1}\right)^{*}\left[\lambda r_{2}\right]\right)+\lambda r_{2}\left(\alpha \gamma \mu_{2}\left(n-a^{*}\left[\lambda r_{2}\right]\left(-1+v^{*}\left[\lambda r_{1}\right]\right)(-1+\right.\right.$ $\left.\left.n+v^{*}\left[\lambda r_{2}\right]\right)\left(h_{1}\right)^{*}\left[\lambda r_{2}\right]\left(1-q+q\left(u_{1}\right)^{*}\left[\lambda r_{2}\right]\right)\right)+\mu_{1}(n \alpha \gamma+$ $\mu_{2}\left(2 n \alpha+\gamma+a^{*}\left[\lambda r_{2}\right]\left(-\alpha-\gamma+\alpha v^{*}\left[\lambda r_{1}\right]\right)(-1+n+\right.$ $\left.\left.\left.\left.\left.\left.v^{*}\left[\lambda r_{2}\right]\right)\left(h_{1}\right)^{*}\left[\lambda r_{2}\right]\left(-1+q-q\left(u_{1}\right)^{*}\left[\lambda r_{2}\right]\right)\right)\right)\right)\right)\right\}$,

and

$n=\left(\frac{1}{a^{*}\left[\lambda r_{1}\right]\left(h_{2}\right)^{*}\left[\lambda r_{1}\right]\left(1-q+q\left(u_{2}\right)^{*}\left[\lambda r_{1}\right]\right)}\right)$.

Hence, the steady-state availability of the system can be given as

$\mathrm{Av}=P_{0}+P_{1}+P_{2}+P_{4}+P_{6}+P_{7}+P_{10}+P_{11}+P_{13}+P_{14}$

$\mathrm{Av}=\frac{c_{0}}{\lambda r_{1} r_{2}}\left(r_{1} a^{*}\left[\lambda r_{2}\right]\left(-1+n+v^{*}\left[\lambda r_{2}\right]\right)\left(h_{1}\right)^{*}\left[\lambda r_{2}\right](1-q+\right.$ $\left.q\left(u_{1}\right)^{*}\left[\lambda r_{2}\right]\right)+r_{2}\left(1+\lambda r_{1}\left(\bar{V}^{*}\left[\lambda r_{2}\right]+(-1+n+\right.\right.$ $\left.v^{*}\left[\lambda r_{2}\right]\right) \bar{A}^{*}\left[\lambda r_{2}\right]\left(h_{1}\right)^{*}\left[\lambda r_{2}\right]+n \bar{A}^{*}\left[\lambda r_{1}\right]\left(h_{2}\right)^{*}\left[\lambda r_{1}\right]-$ $\left(H_{1}\right)^{*}\left[\lambda r_{2}\right]+n\left(H_{1}\right)^{*}\left[\lambda r_{2}\right]+v^{*}\left[\lambda r_{2}\right]\left(H_{1}\right)^{*}\left[\lambda r_{2}\right]+$ $n\left(H_{2}\right)^{*}\left[\lambda r_{1}\right]+a^{*}\left[\lambda r_{2}\right](-1+n+$ $\left.v^{*}\left[\lambda r_{2}\right]\right)\left(h_{1}\right)^{*}\left[\lambda r_{2}\right]\left(\bar{V}^{*}\left[\lambda r_{1}\right]\left(1-q+q\left(u_{1}\right)^{*}\left[\lambda r_{2}\right]\right)+\right.$ $\left.\left.\left.\left.q\left(\bar{U}_{1}\right)^{*}\left[\lambda r_{2}\right]\right)+n q a^{*}\left[\lambda r_{1}\right]\left(h_{2}\right)^{*}\left[\lambda r_{1}\right]\left(\bar{U}_{2}\right)^{*}\left[\lambda r_{1}\right]\right)\right)\right)$,

\section{RELIABILITY OF THE SYSTEM}

Making use the method similar to that in Sec. (4) , the following partial-differential equations can be obtained:

$$
\begin{aligned}
\left(\frac{d}{d t}+r_{1} \lambda\right) L_{0}(t)= & \int_{0}^{\infty} k_{2}\left(u_{2}\right) L_{14}\left(t, u_{2}\right) d u_{2} \\
& +\int_{0}^{\infty}(1-q) \gamma(z) L_{13}(t, z) d z, \\
\left(\frac{\partial}{\partial t}+\frac{\partial}{\partial x}+\alpha(x)+\right. & \left.r_{2} \lambda\right) L_{1}(t, x)=0,
\end{aligned}
$$

$$
\begin{aligned}
& \left(\frac{\partial}{\partial t}+\frac{\partial}{\partial y_{1}}+\mu_{1}\left(y_{1}\right)+r_{2} \lambda\right) L_{2}\left(t, y_{1}\right)=0, \\
& \left(\frac{\partial}{\partial t}+\frac{\partial}{\partial z}+\gamma(z)+r_{2} \lambda\right) L_{4}(t, z)=0, \\
& \left(\frac{d}{d t}+r_{2} \lambda\right) L_{6}(t)=\int_{0}^{\infty} k_{1}\left(u_{1}\right) L_{7}\left(t, u_{1}\right) d u_{1} \\
& \quad+\int_{0}^{\infty}(1-q) \gamma(z) L_{4}(t, z) d z, \\
& \left(\frac{\partial}{\partial t}+\frac{\partial}{\partial u_{1}}+k_{1}\left(u_{1}\right)+r_{2} \lambda\right) L_{7}\left(t, u_{1}\right)=0, \\
& \left(\frac{\partial}{\partial t}+\frac{\partial}{\partial y_{2}}+\mu_{2}\left(y_{2}\right)+r_{1} \lambda\right) L_{10}\left(t, y_{2}\right)=0, \\
& \left(\frac{\partial}{\partial t}+\frac{\partial}{\partial x}+\alpha(x)+r_{1} \lambda\right) L_{11}(t, x)=0, \\
& \left(\frac{\partial}{\partial t}+\frac{\partial}{\partial z}+\gamma(z)+r_{1} \lambda\right) L_{13}(t, z)=0, \\
& \text { and, }\left(\frac{\partial}{\partial t}+\frac{\partial}{\partial u_{2}}+k_{2}\left(u_{2}\right)+r_{1} \lambda\right) L_{14}\left(t, u_{2}\right)=0,
\end{aligned}
$$

Their boundary conditions are:-

$$
\begin{aligned}
& L_{1}(t, 0)=r_{1} \lambda L_{0}(t)+\delta(t), \\
& L_{2}(t, 0)=\int_{0}^{\infty} \alpha(x) L_{1}(t, x) d x, \\
& L_{4}(t, 0)=\int_{0}^{\infty} \mu_{1}\left(y_{1}\right) L_{2}\left(t, y_{1}\right) d y_{1}, \\
& L_{7}(t, 0)=\int_{0}^{\infty} q \gamma(z) L_{4}(t, z) d z, \\
& L_{10}(t, 0)=\int_{0}^{\infty} \alpha(x) L_{11}(t, x) d x, \\
& L_{11}(t, 0)=r_{2} \lambda L_{6}(t), \\
& L_{13}(t, 0)=\int_{0}^{\infty} \mu_{2}\left(y_{2}\right) L_{10}\left(t, y_{2}\right) d y_{2}, \\
& L_{14}(t, 0)=\int_{0}^{\infty} q \gamma(z) L_{13}(t, z) d z,
\end{aligned}
$$

The initial conditions are

$$
L_{1}(0, x)=\delta(x)= \begin{cases}1, & x=0 \\ 0, & x \neq 0\end{cases}
$$

Otherwise is 0

Taking Laplace transform with respect to $t$ to the equations(85-103), and solving for

$$
L_{i}{ }^{*}(s) ;(i=0,1,2,4,6,7,10,11,13,14) .
$$

We find,

$L_{0}^{*}(s)=\frac{C_{1}}{\lambda r_{1}}$,

$L_{1}{ }^{*}(s, x)=e^{-x\left(s+\lambda r_{2}\right)} C_{1} \bar{V}[x]$,

$L_{2}^{*}(s, x)=e^{-x\left(s+\lambda r_{2}\right)} C_{1} \bar{H}_{1}[x] v^{*}\left[s+\lambda r_{2}\right]$,

$L_{4}{ }^{*}(s, x)=C_{1} e^{-s x-x \lambda r_{2}} \bar{A}[x] v^{*}\left[s+\lambda r_{2}\right]\left(h_{1}\right)^{*}\left[s+\lambda r_{2}\right]$,

$L_{6}{ }^{*}(s)=C_{1} \frac{a^{*}\left[s+\lambda r_{2}\right] v^{*}\left[s+\lambda r_{2}\right]\left(h_{1}\right)^{*}\left[s+\lambda r_{2}\right]\left(1-q+q\left(u_{1}\right)^{*}\left[s+\lambda r_{2}\right]\right)}{s+\lambda r_{2}}$,

(108)

$L_{7}{ }^{*}(s, x)=C_{1} \mathbb{e}^{-s x-x \lambda r_{2}} q \bar{U}_{1}[x] a^{*}\left[s+\lambda r_{2}\right] v^{*}[s+$ $\left.\lambda r_{2}\right]\left(h_{1}\right)^{*}\left[s+\lambda r_{2}\right]$,

$L_{10}{ }^{*}(s, x)=C_{1} \frac{1}{s+\lambda r_{2}} e^{-x\left(s+\lambda r_{1}\right)} \lambda r_{2} \bar{H}_{2}[x] a^{*}\left[s+\lambda r_{2}\right] v^{*}[s+$ $\left.\lambda r_{1}\right] v^{*}\left[s+\lambda r_{2}\right]\left(h_{1}\right)^{*}\left[s+\lambda r_{2}\right]\left(1-q+q\left(u_{1}\right)^{*}\left[s+\lambda r_{2}\right]\right)$,

(110) 
$L_{11}{ }^{*}(s, x)=$

$C_{1} \frac{e^{-x\left(s+\lambda r_{1}\right)} \lambda r_{2} \bar{V}[x] a^{*}\left[s+\lambda r_{2}\right] v^{*}\left[s+\lambda r_{2}\right]\left(h_{1}\right)^{*}\left[s+\lambda r_{2}\right]\left(1-q+q\left(u_{1}\right)^{*}\left[s+\lambda r_{2}\right]\right)}{s+\lambda r_{2}}$

(111)

$L_{13}{ }^{*}(s, x)=C_{1} \frac{1}{s+\lambda r_{2}} e^{-x\left(s+\lambda r_{1}\right)} \lambda r_{2} \bar{A}[x] a^{*}\left[s+\lambda r_{2}\right] v^{*}[s+$

$\left.\lambda r_{1}\right] v^{*}\left[s+\lambda r_{2}\right]\left(h_{1}\right)^{*}\left[s+\lambda r_{2}\right]\left(h_{2}\right)^{*}\left[s+\lambda r_{1}\right](1-q+$

$\left.q\left(u_{1}\right)^{*}\left[s+\lambda r_{2}\right]\right)$,

$L_{14}{ }^{*}(s, x)=C_{1} \frac{1}{s+\lambda r_{2}} e^{-x\left(s+\lambda r_{1}\right)} q \lambda r_{2} \bar{U}_{2}[x] a^{*}\left[s+\lambda r_{1}\right] a^{*}[s+$ $\left.\lambda r_{2}\right] v^{*}\left[s+\lambda r_{1}\right] v^{*}\left[s+\lambda r_{2}\right]\left(h_{1}\right)^{*}\left[s+\lambda r_{2}\right]\left(h_{2}\right)^{*}\left[s+\lambda r_{1}\right](1-$ $\left.q+q\left(u_{1}\right)^{*}\left[s+\lambda r_{2}\right]\right)$,

Where, $C_{l}$ :-

$$
\begin{aligned}
C_{1}^{-1}=1-\frac{1}{\left(s+\lambda r_{1}\right)\left(s+\lambda r_{2}\right)} \lambda^{2} r_{1} r_{2} a^{*}\left[s+\lambda r_{1}\right] a^{*}[s \\
\left.+\lambda r_{2}\right] v^{*}\left[s+\lambda r_{1}\right] v^{*}\left[s+\lambda r_{2}\right]\left(h_{1}\right)^{*}[s \\
\left.+\lambda r_{2}\right]\left(h_{2}\right)^{*}\left[s+\lambda r_{1}\right]\left(1-q+q\left(u_{1}\right)^{*}[s\right. \\
\left.\left.+\lambda r_{2}\right]\right)\left(1-q+q\left(u_{2}\right)^{*}\left[s+\lambda r_{1}\right]\right)
\end{aligned}
$$

Since, the reliability of the system is

$$
\begin{aligned}
R(t) & =L_{0}(t)+\int_{0}^{\infty} L_{1}(\mathrm{t}, \mathrm{x}) \mathrm{dx}+\int_{0}^{\infty} L_{2}(\mathrm{t}, \mathrm{x}) \mathrm{dx} \\
& +\int_{0}^{\infty} L_{4}(\mathrm{t}, \mathrm{x}) \mathrm{dx}+L_{6}(\mathrm{t})+\int_{0}^{\infty} L_{7}(\mathrm{t}, \mathrm{x}) \mathrm{dx}+\int_{0}^{\infty} L_{10}(\mathrm{t}, \mathrm{x}) \mathrm{dx} \\
& +\int_{0}^{\infty} L_{11}(\mathrm{t}, \mathrm{x}) \mathrm{dx}+\int_{0}^{\infty} L_{13}(\mathrm{t}, \mathrm{x}) \mathrm{dx}+\int_{0}^{\infty} L_{14}(\mathrm{t}, \mathrm{x}) \mathrm{dx}
\end{aligned}
$$

Then,

$$
\begin{aligned}
R^{*}(s) & =L_{0}{ }^{*}(s)+\int_{0}^{\infty} L_{1}{ }^{*}(\mathrm{~s}, \mathrm{x}) \mathrm{dx}+\int_{0}^{\infty} L_{2}{ }^{*}(\mathrm{~s}, \mathrm{x}) \mathrm{dx} \\
& +\int_{0}^{\infty} L_{4}{ }^{*}(\mathrm{~s}, \mathrm{x}) \mathrm{dx}+L_{6}{ }^{*}(\mathrm{~s})+\int_{0}^{\infty} L_{7}{ }^{*}(\mathrm{~s}, \mathrm{x}) \mathrm{dx} \\
& +\int_{0}^{\infty} L_{10}{ }^{*}(\mathrm{~s}, \mathrm{x}) \mathrm{dx}+\int_{0}^{\infty} L_{11}{ }^{*}(\mathrm{~s}, \mathrm{x}) \mathrm{dx}+\int_{0}^{\infty} L_{13}{ }^{*}(\mathrm{~s}, \mathrm{x}) \mathrm{dx} \\
& +\int_{0}^{\infty} L_{14}{ }^{*}(\mathrm{~s}, \mathrm{x}) \mathrm{dx}
\end{aligned}
$$

which lead to

$$
\begin{aligned}
& R^{*}(s)=C_{1}\left(\bar{V}^{*}\left[s+\lambda r_{2}\right]+\frac{1}{\left(s+\lambda r_{1}\right)\left(s+\lambda r_{2}\right)} v^{*}\left[s+\lambda r_{2}\right]((s+\right. \\
& \left.\lambda r_{1}\right)\left(s+\lambda r_{2}\right)\left(\bar{A}^{*}\left[s+\lambda r_{2}\right]\left(h_{1}\right)^{*}\left[s+\lambda r_{2}\right]+\left(\bar{H}_{1}\right)^{*}[s+\right. \\
& \left.\left.\lambda r_{2}\right]\right)+a^{*}\left[s+\lambda r_{2}\right]\left(h_{1}\right)^{*}\left[s+\lambda r_{2}\right]\left(s-q s+q s\left(\left(u_{1}\right)^{*}[s+\right.\right. \\
& \left.\left.\lambda r_{2}\right]+s\left(\bar{U}_{1}\right)^{*}\left[s+\lambda r_{2}\right]\right)+\lambda r_{2}\left(s \bar{V}^{*}\left[s+\lambda r_{1}\right](1-q+\right. \\
& \left.q\left(u_{1}\right)^{*}\left[s+\lambda r_{2}\right]\right)+s v^{*}\left[s+\lambda r_{1}\right]\left(1-q+q\left(u_{1}\right)^{*}[s+\right. \\
& \left.\left.\lambda r_{2}\right]\right)\left(\bar{A}^{*}\left[s+\lambda r_{1}\right]\left(h_{2}\right)^{*}\left[s+\lambda r_{1}\right]+\left(\bar{H}_{2}\right)^{*}\left[s+\lambda r_{1}\right]\right)+ \\
& q s\left(\bar{U}_{1}\right)^{*}\left[s+\lambda r_{2}\right]+a^{*}\left[s+\lambda r_{1}\right] v^{*}\left[s+\lambda r_{1}\right]\left(h_{2}\right)^{*}[s+ \\
& \left.\lambda r_{1}\right]\left(1-q+q\left(u_{1}\right)^{*}\left[s+\lambda r_{2}\right]\right)\left(1-q+q\left(u_{2}\right)^{*}\left[s+\lambda r_{1}\right]+\right. \\
& \left.\left.q s\left(\bar{U}_{2}\right)^{*}\left[s+\lambda r_{1}\right]\right)\right)+\lambda r_{1}\left(1-q+q\left(u_{1}\right)^{*}\left[s+\lambda r_{2}\right]+\right. \\
& q s\left(\bar{U}_{1}\right)^{*}\left[s+\lambda r_{2}\right]+\lambda r_{2}\left(\overline { V } ^ { * } [ s + \lambda r _ { 1 } ] \left(1-q+q\left(u_{1}\right)^{*}[s+\right.\right. \\
& \left.\left.\lambda r_{2}\right]\right)+q\left(\bar{U}_{1}\right)^{*}\left[s+\lambda r_{2}\right]+v^{*}\left[s+\lambda r_{1}\right]\left(1-q+q\left(u_{1}\right)^{*}[s+\right. \\
& \left.\left.\lambda r_{2}\right]\right)\left(\left(\bar{H}_{2}\right)^{*}\left[s+\lambda r_{1}\right]+\left(h_{2}\right)^{*}\left[s+\lambda r_{1}\right]\left(\bar{A}^{*}\left[s+\lambda r_{1}\right]+\right.\right. \\
& \left.\left.\left.\left.\left.\left.\left.q a^{*}\left[s+\lambda r_{1}\right]\left(\bar{U}_{2}\right)^{*}\left[s+\lambda r_{1}\right]\right)\right)\right)\right)\right)\right)\right)
\end{aligned}
$$

The mean time to the system failure (MTTF) can be calculated on:

$M T T F=R^{*}(0)=$

$\left(\bar{V}^{*}\left[\lambda r_{2}\right]+\frac{1}{\lambda^{2} r_{1} r_{2}} v^{*}\left[\lambda r_{2}\right]\left(\lambda^{2} r_{1} r_{2}\left(\bar{A}^{*}\left[\lambda r_{2}\right]\left(h_{1}\right)^{*}\left[\lambda r_{2}\right]+\right.\right.\right.$
$\left.\left(\bar{H}_{1}\right)^{*}\left[\lambda r_{2}\right]\right)+$

$a^{*}\left[\lambda r_{2}\right]\left(h_{1}\right)^{*}\left[\lambda r_{2}\right]\left(\lambda r_{2} a^{*}\left[\lambda r_{1}\right] v^{*}\left[\lambda r_{1}\right]\left(h_{2}\right)^{*}\left[\lambda r_{1}\right](1-q+\right.$ $\left.q\left(u_{1}\right)^{*}\left[\lambda r_{2}\right]\right)\left(1-q+q\left(u_{2}\right)^{*}\left[\lambda r_{1}\right]\right)+\lambda r_{1}(1-q+$ $q\left(u_{1}\right)^{*}\left[\lambda r_{2}\right]+\lambda r_{2}\left(\bar{V}^{*}\left[\lambda r_{1}\right]\left(1-q+q\left(u_{1}\right)^{*}\left[\lambda r_{2}\right]\right)+\right.$ $q\left(\bar{U}_{1}\right)^{*}\left[\lambda r_{2}\right]+v^{*}\left[\lambda r_{1}\right]\left(1-q+q\left(u_{1}\right)^{*}\left[\lambda r_{2}\right]\right)\left(\left(\bar{H}_{2}\right)^{*}\left[\lambda r_{1}\right]+\right.$ $\left.\left.\left.\left.\left.\left.\left(h_{2}\right)^{*}\left[\lambda r_{1}\right]\left(\bar{A}^{*}\left[\lambda r_{1}\right]+q a^{*}\left[\lambda r_{1}\right]\left(\bar{U}_{2}\right)^{*}\left[\lambda r_{1}\right]\right)\right)\right)\right)\right)\right)\right) /(1-$ $a^{*}\left[\lambda r_{1}\right] a^{*}\left[\lambda r_{2}\right] v^{*}\left[\lambda r_{1}\right] v^{*}\left[\lambda r_{2}\right]\left(h_{1}\right)^{*}\left[\lambda r_{2}\right]\left(h_{2}\right)^{*}\left[\lambda r_{1}\right](1-q+$ $\left.\left.q\left(u_{1}\right)^{*}\left[\lambda r_{2}\right]\right)\left(1-q+q\left(u_{2}\right)^{*}\left[\lambda r_{1}\right]\right)\right)$,

(116)

\section{SPECIAL CASE}

Studying the following special cases:

- Case 1: if $\left(r_{1}=r_{2}=0\right)$, then it means that shocks do not impair on the working unit and the units will never fail.

- Case2: if $\left(r_{1}=r_{2}=1\right)$ and $\{P(X=0)=1\}$, then each shock will cause the working unit to fail and the system becomes un-repairable system.

- Case3: if $\left(r_{1}=r_{2}=1\right)$ and $\{q=0\}$, then each shock will cause the working unit to fail and the units do not need post repaired.

\section{NUMERICAL EXAMPLE AND STUDY OF SYSTEM BEHAVIOR THROUGH GRAPHS}

Let $\alpha(x)=\alpha, \gamma(z)=\gamma, \mu_{i}\left(y_{i}\right)=\mu_{i}$ and $\mathrm{k}_{\mathrm{i}}\left(\mathrm{t}_{\mathrm{i}}\right)=\mathrm{k}_{\mathrm{i}}$; where $(i=1,2)$.

We plot the steady- state availability and mean time to system failure for the system model. We show that

- In Fig (1), the steady- state availability is decreasing if the probabilities that one shock causes unit 1,2 to fail $\left(r_{1}, r_{2}\right)$ are increasing.

- In Fig(2), the steady- state availability is increasing if the repair rate of unit $1,2\left(\mu_{1}, \mu_{2}\right)$ are increasing.

- In Fig(3), the steady- state availability is increasing if the recall repairman rate $\alpha$, and inspection rate $\gamma$ are increasing.

- In Fig(4), the steady- state availability is increasing if the post repair rate of unit1,2( $\left.k_{1}, k_{2}\right)$ are increasing.

- In Fig (5), the mean time to system failure is decreasing if the probabilities that one shock causes unit 1,2 to fail $\left(r_{1}, r_{2}\right)$ are increasing.

- In Fig(6), the mean time to system failure is increasing if the repair rate of unit $1,2\left(\mu_{1}, \mu_{2}\right)$ are increasing.

- In Fig(7), the mean time to system failure is increasing if the recall repairman rate $\alpha$, and inspection rate $\gamma$ are increasing.

- In Fig(8), the mean time to system failure is increasing if the post repair rate of unit $1,2\left(k_{1}, k_{2}\right)$ are increasing. 


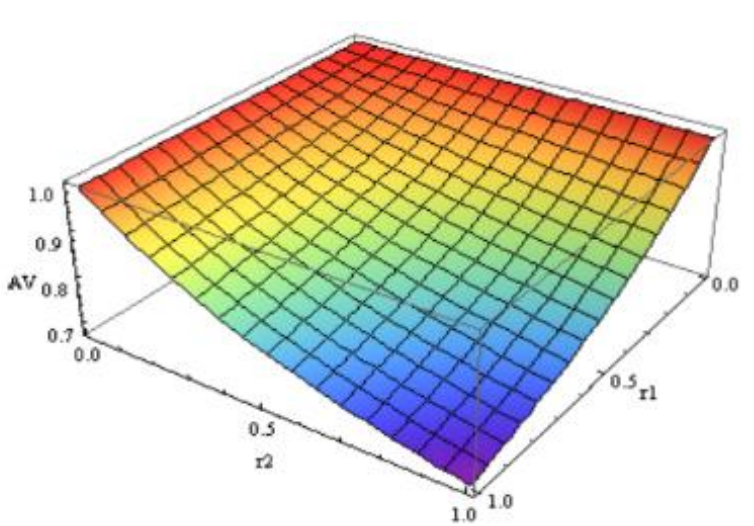

Figure (1)

S.S.Availabilty vs $\left(r_{1}, r_{2}\right)$

where $\left(\mu_{1}=0.3, \mu_{2}=0.4, \gamma=0.8, k_{1}=0.7, k_{2}=0.6, \alpha=\right.$ $0.9, q=0.5$ and $\lambda=0.2$ )

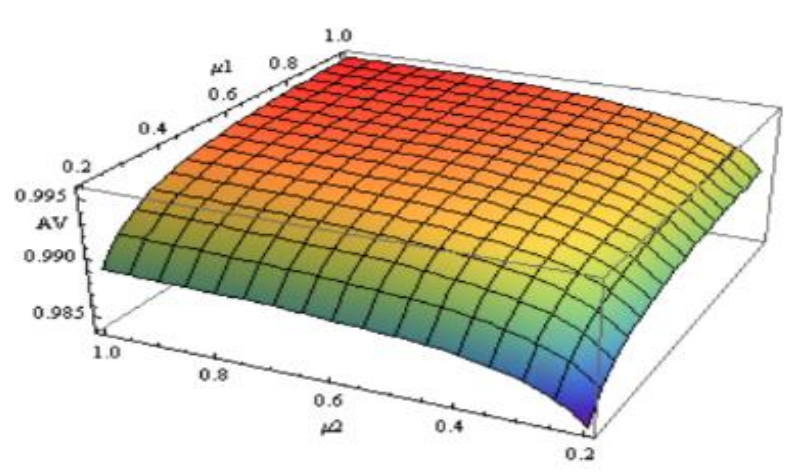

Figure (2)

S.S.Availabilty vs. repair rate of unit $1,2\left(\mu_{1}, \mu_{2}\right)$

Where $\left(r_{1}=0.1, r_{2}=0.1, \lambda=0.2, \gamma=0.8, k_{1}=0.7, k_{2}=\right.$ $0.6, \alpha=0.9$ and $q=0.5$ ).

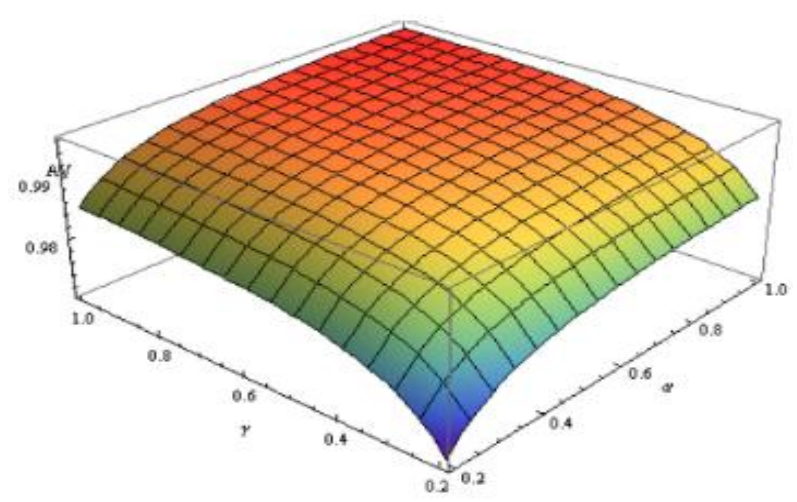

Figure (3)

S.S.Availabilty vs. recall repairman rate $\alpha$,and inspection rate $\gamma$.

Where $\left(r_{1}=0.1, r_{2}=0.1, \lambda=0.2, \mu_{1}=0.8, \mu_{2}=0.9, k_{1}=\right.$ $0.7, k_{2}=0.6$ and $q=0.5$ ).

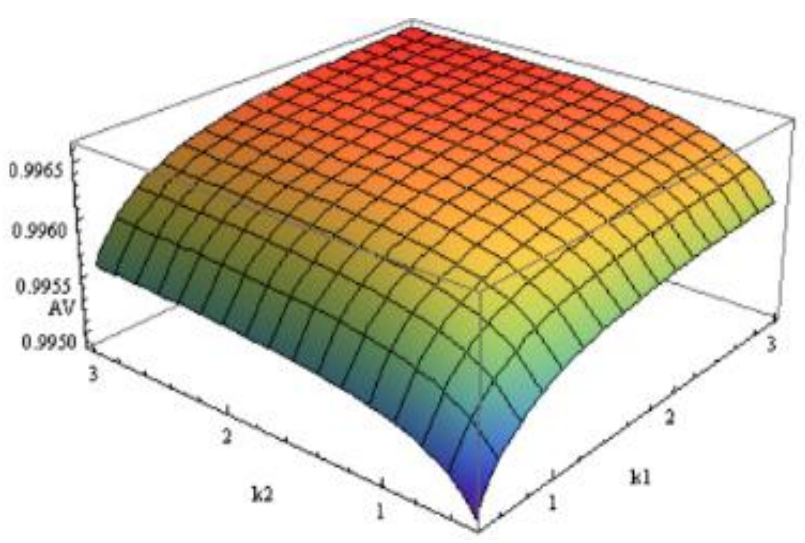

Figure (4)

S.S.Availabilty vs. post repair rate of unit $1,2\left(k_{1}, k_{2}\right)$

Where $\left(r_{1}=0.1, r_{2}=0.1, \lambda=0.2, \mu_{1}=0.8, \mu_{2}=0.9, \gamma=\right.$ $0.8, \alpha=0.9$ and $q=0.5)$.

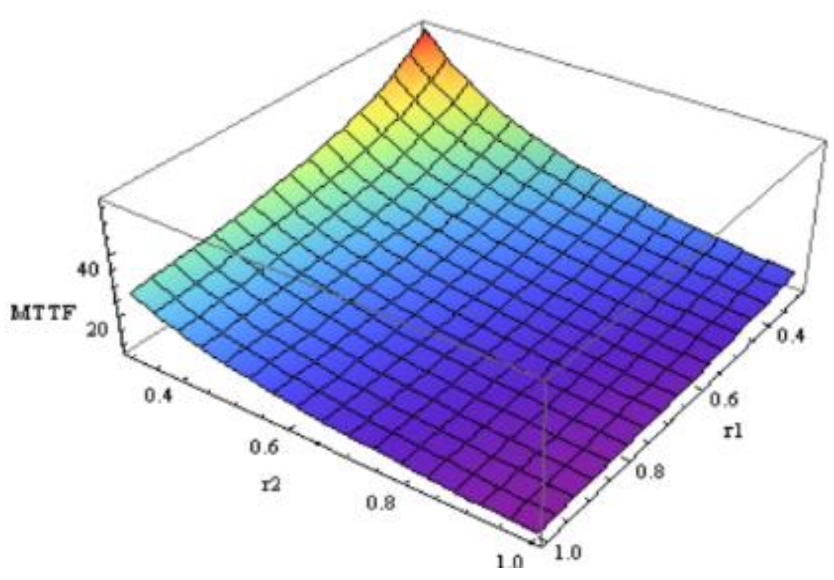

Figure (5)

MTTF vs $\left(r_{1}, r_{2}\right)$

where $\left(\mu_{1}=0.3, \mu_{2}=0.4, \gamma=0.8, k_{1}=0.7, k_{2}=0.6, \alpha=\right.$ $0.9, q=0.5$ and $\lambda=0.2$ ).

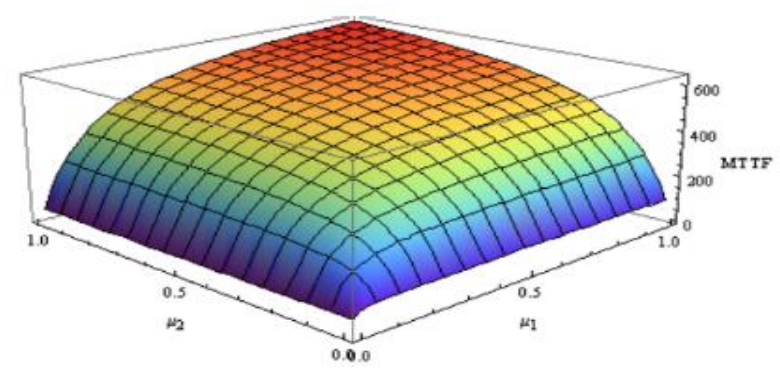

Figure (6)

MTTF vs. repair rate of unit1,2( $\left.\mu_{1}, \mu_{2}\right)$

where $\left(r_{1}=0.1, r_{2}=0.1, \lambda=0.2, \gamma=0.8, k_{1}=0.7, k_{2}=\right.$ $0.6, \alpha=0.9$ and $q=0.5$ ). 


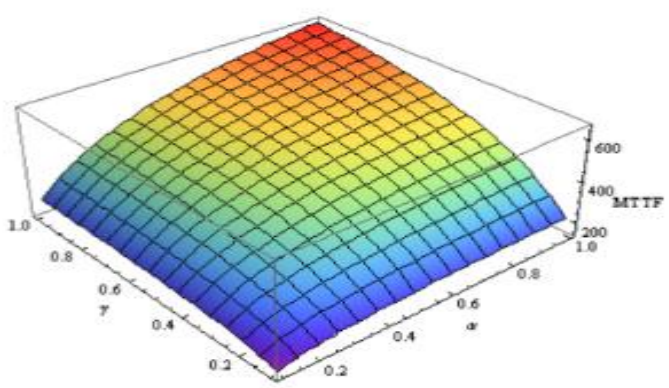

Figure (7)

MTTF vs. recall repairman rate $\alpha$, and inspection rate $\gamma$.

Where $\left(r_{1}=0.1, r_{2}=0.1, \lambda=0.2, \mu_{1}=0.8, \mu_{2}=0.9, k_{1}=\right.$ $0.7, k_{2}=0.6$ and $q=0.5$ ).

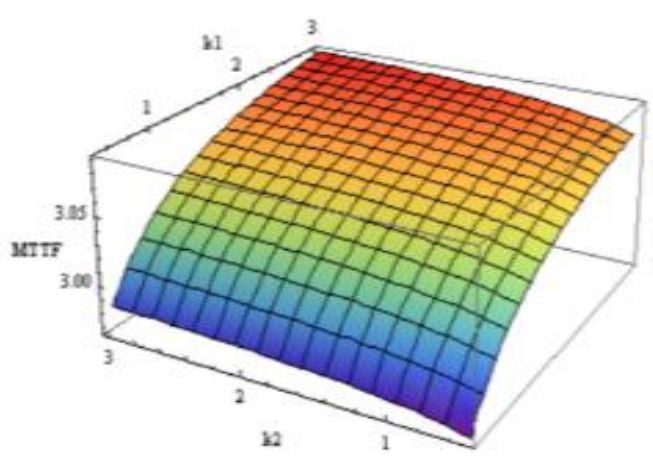

Figure (8)

MTTF vs. post repair rate of unit1,2( $\left.k_{1}, k_{2}\right)$

Where $\left(r_{1}=0.1, r_{2}=0.1, \lambda=0.2, \mu_{1}=0.8, \mu_{2}=0.9, \gamma=\right.$ $0.8, \alpha=0.9$ and $q=0.5$ ).

\section{CONCLUSIONS}

In this paper some reliability measures of the system such as, the mean time to system failure (MTTF) and the steady state availability of the system under Poisson shocks with inspection and post repair using the supplementary variable technique and Laplace transform are successfully obtained. The followings are noticed

1. The MTTF and $A v(\infty)$ decrease by increasing of $r_{1}$ and $r_{2}$.

2. The MTTF and $A v(\infty)$ increase by increasing of $\mu_{1}$ and $\mu_{2}$

3. The MTTF and $A v(\infty)$ increase by increasing of $\alpha$ and $\gamma$.

4. The MTTF and $A v(\infty)$ increase by increasing of $k_{1}$ and $k_{2}$.

Based on the results obtained for a particular case, it is concluded that the system model can be more reliable and profitable to use by increasing the repair rates, recall repair repairman rate, inspection rate and post repair rates. The system can be in its best condition when the probabilities $r_{1}, r_{2}$ decrease.

\section{ACKNOWLEDGMENTS}

The authors would like to thank the referees for their valuable comments that improved the presentation of this article.

\section{REFERENCES}

[1] Abdul Ameer Al-Ali and Murari K. One unit reliability system subject to random shocks and preventive maintenance. J.microelectron. Reliab., 28(3):373-377, 1988.

[2] Shengqiang Chen, Xianyun Meng, and Yutian Chen. Analysis of a cold standby system with an unreliable repair facility and vacation under poisson shocks. Journal of Information \& Computational Science, 11(4):1217$1224,2014$.

[3] S. K. Chhillar, S. C. Malik, and J. Nandal. Analysis of a system reliability model subject to degradation and random shocks. International Journal of Statistics and Applications, 3(3):64-70, 2013.

[4] Ahmed Khayar. Coast analysis of two-sililar-unit cold standby system with three states under human failure using first order linear differential equations. Pinnacle Engineering \& Technology, 2(2):1-8, 2014.

[5] Renbin Lin, Zaiming Liu, and Yong Wu. Analysis of a repairable system with an unreliable repair facility and a single vacation. journal of information \& computational science, 8(10):1949-1957, 2011.

[6] Haitao liu, Xianyn meng, and Wenjuan Wu. The cold standby system with repair of non-new and repairman vacation. Journal of Computational Information Systems, 8(4):1349-1357, 2012.

[7] Wenjuan LIU, Xianyun MENG, Xinxin ZHANG, Shengqiang CHEN, and Lijiao TAN. A cold standby repairable deteriorating system of three components with repairman vacation and priority. Journal of Computational Information Systems, 9(10):4031-4039, 2013.

[8] M.A.W Mahmoud and M.E. Moshref. On a two-unit cold standby system considering hardware, human error failures and preventive maintenance. Mathematical and computer modelling, 51(5-6):736-745, 2010.

[9] Yangli Ren, Lina Guo, and Lingling Zhang. Stability analysis of a repairable system with warning device and repairman vacation. Journal of Function Spaces and Applications, 2013(Article ID 674191):19 pages, 2013.

[10] Q. T. Wu and S. M. Wu. Reliability analysis of two-unit cold standby repairable systems under poisson shocks. Applied Mathematics and Computation, 218(1):171-182, 2011.

[11] Qingtai Wu. Reliability analysis of a cold standby system attacked by shocks. Applied Mathematics and Computation, 218:11654-11673, 2012.

[12] L. Yuan. Reliability analysis for a k-out-of-n:g system with redundant dependency and repairmen having multiple vacations. Applied Matheamtics and Computation, 218(24):11959-11969, 2012.

[13] L. Yuan and J. Xu. A deteriorating system with its repairman having multiple vacations. Applied Mathematics and Computation, 217(10):4980-4989, 2011.

[14] W. Z. Yuan and G. Q. Xu. Modelling of a deteriorating system with repair satisfying general distribution. Applied Mathematics and Computation, 218(11):6340 6350, 2012. 